\title{
Magnetic excitations in Dy/Y superlattices as seen via inelastic neutron scattering
}

\author{
A. T. D. Grünwald, ${ }^{1}$ A. R. Wildes, ${ }^{2, *}$ W. Schmidt, ${ }^{3}$ E. V. Tartakovskaya, ${ }^{1,4}$ J. Kwo, ${ }^{5}$ C. Majkrzak, ${ }^{6}$ R. C. C. Ward, ${ }^{7}$ and \\ A. Schreyer ${ }^{1}$ \\ ${ }^{1}$ GKSS Research Centre, 21502 Geesthacht, Germany \\ ${ }^{2}$ Institut Laue-Langevin, 38042 Grenoble, France \\ ${ }^{3}$ IFF, JCNS at ILL, Forschungszentrum Jülich, 38042 Grenoble, France \\ ${ }^{4}$ Institute for Magnetism of National Ukrainian Academy of Science, 03142 Kiev, Ukraine \\ ${ }^{5}$ Department of Physics, National Tsing Hua University, 300 Hsinchu, Taiwan \\ ${ }^{6}$ NIST Center for Neutron Research, National Institute of Standards and Technology, Gaithersburg, Maryland 20899, USA \\ ${ }^{7}$ Clarendon Laboratory, Oxford University, South Parks Rd, Oxford OX1 3PU, United Kingdom \\ (Received 14 January 2010; revised manuscript received 27 April 2010; published 23 July 2010)
}

\begin{abstract}
Measurements of the spin excitations propagating normal to the interfaces in Dy/Y superlattices using neutron inelastic scattering are presented. For a given magnon momentum, a neutron-scattering spectrum shows multiple peaks at different energies, which indicates discrete energy spectra. The results are compared with theoretical calculations developed here to describe magnetic excitations in rare-earth superlattices. The theory accounts for Ruderman-Kittel-Kasuya-Yosida (RKKY) and Dzyaloshinsky-Moriya interactions in incommensurate helicoidal structures and achieves a quantitative agreement with the experimental data. This work demonstrates that neutron inelastic scattering can be used for systematic studies of the exchange interactions and spin dynamics in nanomagnetic systems over wide areas of the Brillouin zone.
\end{abstract}

DOI: 10.1103/PhysRevB.82.014426

PACS number(s): 75.30.Ds, 73.21.Cd, 78.70.Nx

\section{INTRODUCTION}

Many of the rare-earths have complicated magnetic structures and phase diagrams. This is remarkable considering that their magnetism is due to localized moments and the mediation can be given by simple (RKKY) interactions. Bulk materials were the subject of intense scrutiny during the 1980s and 1990s, where rare-earth metals and alloys proved to be invaluable model systems for the study of magnetism. ${ }^{1,2}$ Interest intensified with the discovery that epitaxial superlattice samples could be grown, where a bilayer could be repeatedly stacked without losing crystal coherence. ${ }^{3}$ Modulation in the magnetic properties and long range, coherent spin structures were observed when the superlattice periodicity was comparable to the RKKY interaction range. ${ }^{4,5}$ Recent work suggests that the DzyaloshinskyMoriya interaction (DMI) is also important in helimagnetic rare-earth superlattices. ${ }^{6}$

The key to understanding magnetic behavior lies in the dynamics of the magnetic lattice. Neutron inelastic scattering has proven to be an indispensable tool for such studies. It is the only known experimental tool that is able to measure lattice excitations across the entire Brillouin zone. Neutron studies of the magnetic dynamics can therefore give a comprehensive explanation for why a material has its magnetic properties, as has proved to be the case in the investigation of bulk rare earths. ${ }^{1}$ When applied to small sample masses, however, the technique suffers from low count rates. Thus, to date, there have been no concerted efforts to apply the technique to superlattices whose masses are much less than a gramme.

This article reports the successful use of neutron inelastic scattering to measure the spin excitations along the interface normal direction in a Dy/Y superlattice. The measurements were carried out at the Institut Laue-Langevin in Grenoble,
France, using the cold neutron three-axis spectrometers IN12 and IN14, and represent a significant advance from preliminary measurements that have been previously published. ${ }^{7}$ In conjunction, a theory that describes the magnetic excitations in the sample is presented. The theory quantitatively, and favorably, is compared with the data.

It has been previously established that, in the ground state, Dy/Y superlattices have an incommensurate helimagnetic structure. ${ }^{4,5}$ In contrast, bulk Dy is ferromagnetic in its ground state but enters a helimagnetic state above $89 \mathrm{~K}$ and below its Néel temperature $(179 \mathrm{~K}) .{ }^{1}$ The phase of the helical magnetic order in the Dy propagates coherently through the nonmagnetic $\mathrm{Y}$ layers and the interaction can be described by RKKY theory. Thus, despite the fact that $\mathrm{Y}$ has negligible magnetism, each $\mathrm{Y}$ monolayer may be regarded as having a virtual moment with a magnitude of zero but a direction propagating the phase of the helicity. The helical wave vector increases with increasing temperature. A comprehensive discussion on the magnetic structures of Dy/Y superlattices, including the effects of changing the thicknesses of the respective constituents of the bilayer, is presented elsewhere. ${ }^{5}$

\section{THEORY}

A number of theoretical approaches have been proposed to describe the magnetic excitations in multilayers, rare earth, and otherwise. ${ }^{1,8}$ The approach used here adapts previous models ${ }^{9-12}$ where the excitations were calculated by considering the interactions between discrete monolayers using linear spin wave theory. This type of model has been shown to be appropriate for heavy rare earths with strong anisotropy, such as Dy, ${ }^{1}$ and gives flexibility when treating both incommensurate structures and the chemical modulation of an artificial superlattice. 
Dy/Y superlattices have a hexagonal close-packed structure and are typically grown with the growth direction, and therefore the chemical modulation, along the [0001] direction. The monolayers considered in the model were therefore the atomic planes along the $c$ axis. The moments in an individual monolayer were regarded as being ferromagnetically coupled with the moments lying in the plane. The ferromagnetic direction rotates about the normal by a turn angle $\phi\left(R_{i}\right)$, where $R_{i}$ is the position of the corresponding monolayer along $c$, resulting in a helimagnetic structure. The turn angle is known to be different ${ }^{4}$ depending on whether the monolayers are $\mathrm{Y}$ or Dy.

The spin-wave dispersion was derived from a standard Heisenberg exchange Hamiltonian with anisotropy and DMI

$$
H=H_{\mathrm{DMI}}+H_{A}-\sum_{i \neq j} J\left(R_{i}-R_{j}\right) \mathbf{S}_{i} \cdot \mathbf{S}_{j},
$$

where $\mathbf{S}_{i}$ is the spin per atom on monolayer $i$ and $\left(R_{i}-R_{j}\right)$ equals the distance between an integer number of monolayers. The exchange function $J\left(R_{i}-R_{j}\right)$ is given by RKKY interactions. ${ }^{1}$

The anisotropy $H_{A}$ was taken to be a strong easy-plane anisotropy

$$
H_{A}=K \sum_{i}\left(S_{i}^{c}\right)^{2},
$$

where $S_{i}^{c}$ is the component of $\mathbf{S}$ along the $c$ axis of monolayer $i$, and $K=0.254 \mathrm{meV}$ is the single-ion anisotropy constant adapted from previous estimates for bulk Dy. ${ }^{13,14}$

The term $H_{\text {DMI }}$ gives the contribution of the DMI. It accounts for the presence of magnetic domains with different chirality in the helimagnetic structure of Dy/Y superlattices. These domains will have an influence on the energies of propagating spin waves. Even in zero applied field, previous experiments show an imbalance in the population of these domains. ${ }^{6}$ The term is defined by

$$
H_{\mathrm{DMI}}=\sum_{i, j} \mathbf{D}_{i j} \cdot\left(\mathbf{S}_{i} \times \mathbf{S}_{j}\right),
$$

where $\mathbf{D}_{i, j}$ is the Dzyaloshinsky-Moriya vector.

The theory used here differs from previous treatments in the way that the exchange function is included, and in the subsequent summation over monolayers. The RKKY interaction is very long-ranged, however previous theories have only considered one $\mathrm{e}^{9,10}$ or two ${ }^{11}$ nearest neighbors in the exchange function. Furthermore, the broken symmetry of a superlattice may influence the magnitudes of the exchange parameters. Some reports suggest that a nonmagnetic spacer in a superlattice may enhance the exchange between magnetic monolayers either side of the spacer, ${ }^{15,16}$ which was not considered in previous theories relevant to Dy/Y superlattices. ${ }^{11}$ In the following theory, the contribution from an arbitrary number of layers with arbitrary magnetic exchange may be properly accounted for.

For the summation, a sample is broken down in to its bilayer constituents. Each bilayer has $N$ monolayers containing magnetic material hence the sample is considered to have $N$ magnetic sublattices. The Hamiltonian can then be expressed as

$$
H=\sum_{\nu=1}^{N} H^{\nu}+\sum_{\nu=1}^{N} \sum_{\nu^{\prime} \neq \nu} H^{\nu, \nu^{\prime}},
$$

where $\nu$ and $\nu^{\prime}$ represent sublattice numbers.

On the application of the Holstein-Primakoff transformation and Fourier transforming, the elements in the Hamiltonian may be further expressed

$$
\begin{gathered}
H^{\nu}=\sum_{q} A_{q}^{\nu} a_{q}^{\nu^{\dagger}} a_{q}^{\nu}+\frac{1}{2} B_{q}^{\nu}\left(a_{q}^{\nu} a_{-q}^{\nu}+a_{q}^{\nu^{\dagger}} a_{-q}^{\nu^{\dagger}}\right), \\
H^{\nu, \nu^{\prime}}=\sum_{q} C_{q}^{\nu, \nu^{\prime}} a_{q}^{\nu \dagger} a_{-q}^{\nu^{\prime} \dagger}+D_{q}^{\nu, \nu^{\prime}} a_{q}^{\nu \dagger} a_{-q}^{\nu^{\prime}},
\end{gathered}
$$

where $q$ is the reduced lattice wavenumber, $a_{q}, a_{q}^{\dagger}$ are the Fourier transforms of the appropriate creation and annihilation operators, and

$$
A_{q}^{\nu}=2 \sum_{\nu} \sqrt{S^{\nu} S^{\nu^{\prime}}} J_{\left|\nu-\nu^{\prime}\right|} \cos \left(\Phi_{\nu \nu^{\prime}}\right)+2 K S^{\nu},
$$

$$
\begin{gathered}
B_{q}^{\nu}=2 K S^{\nu}, \\
C_{q}^{\nu, \nu^{\prime}}=-\sqrt{S^{\nu} S^{\nu^{\prime}}} J_{\left|\nu-\nu^{\prime}\right|}\left[1-\cos \left(\Phi_{\nu \nu^{\prime}}\right)\right] \exp \left[i q \frac{c}{2}\left(\nu-\nu^{\prime}\right)\right], \\
D_{q}^{\nu, \nu^{\prime}}=-\sqrt{S^{\nu} S^{\nu^{\prime}}} J_{\left|\nu-\nu^{\prime}\right|}\left[1+\cos \left(\Phi_{\nu \nu^{\prime}}\right)\right] \exp \left[i q \frac{c}{2}\left(\nu-\nu^{\prime}\right)\right] .
\end{gathered}
$$

The parameter $\Phi_{\nu \nu^{\prime}}$ represents $\phi_{\nu^{\prime}}-\phi_{\nu}$, the total rotation of the moment direction between sublattices $\nu$ and $\nu^{\prime}$. The $c$ lattice parameter is very similar between Dy and $\mathrm{Y}$ and a fixed value of $5.7 \AA$ was taken for the model.

In principle, the exchange parameters, $J_{\left|\nu-\nu^{\prime}\right|}$, should extend almost to infinity, as the RKKY interaction is very longranged. For the current discussion, however, two sets of parameters were used. The parameters previously used in an approximation to model the spin waves for bulk Dy were adopted $^{13}$ for magnetic interactions within a Dy layer. These parameters extend from the first $\left(J_{1}\right)$ to the seventh $\left(J_{7}\right)$ nearest monolayer. The exchange between Dy monolayers that are separated by $\mathrm{Y}$ layers, however, required the introduction of additional parameters. Detailed $a b$ initio calculations, needed to give rigorous theoretical estimates, do not exist. Semiphenomenological models exist ${ }^{15}$ however they consider magnetic layers that are far thinner than those in this study and hence cannot be directly applied. Phenomenologically attributed exchange parameters, $J_{n}^{\prime}$ (cf. Ref. 17) were introduced for those Dy monolayers that are separated by the $\mathrm{Y}$ layer. The choice of using these parameters is not meant as a constraint on the limits for the RKKY interactions, which are effectively of infinite length scales, but are meant to represent the minimum number of nearest neighbors that must be included in the calculations to reproduce the measured spectra.

Many sample-dependent properties may be taken in to account in the subsequent summation. Interdiffusion at inter- 
faces can be modeled in a straightforward manner by reducing the magnitudes of the magnetic moments in the monolayers within an interface region. The values of $\Phi_{\nu \nu^{\prime}}$ will vary dependent on whether the appropriate monolayers are of Dy or of $\mathrm{Y}^{4}$ and this too can be accounted for in the summation.

Diagonalization of the Hamiltonian may be achieved using conventional methods for matrices. In this case, the relevant matrix is of rank $2 N$. The resulting Eigenvalues give the spin wave dispersion. The neutron inelastic structure factor can be subsequently calculated in line with the onemagnon cross section. ${ }^{18}$

An example of the calculated inelastic structure factor for spin waves propagating along the Dy/Y [0001] direction is shown in Fig. 1 as a function of $q$. The example has been calculated for $\mathrm{Dy}_{4.3 \mathrm{~nm}} / \mathrm{Y}_{2.8 \mathrm{~nm}}$, corresponding to the structure of the sample that has measured in this study. For clarity, it does not include the DMI or the temperature-dependent magnon occupation (the Bose factor). The nominal width of the $\mathrm{Y}$ is 10 monolayers and of the Dy is 15 monolayers. Based on fits to diffraction data, the interfaces are assumed to be 4 monolayers thick, and the concentration of Dy on these monolayers follows an error function. Exchange across the $\mathrm{Y}$ hence required the addition of $J_{7}^{\prime}=0.1 \mathrm{meV}$ [cf. $J_{7}=$ $-0.008 \mathrm{meV}$ for bulk Dy (Ref. 13)] and $J_{8}^{\prime}=-0.02 \mathrm{meV}$. These values were refined from comparison with the data and their relatively large magnitude is consistent with the observation that the RKKY interaction is less damped when mediated through Y. ${ }^{15}$ For reference, the measured dispersion for bulk Dy is also shown. ${ }^{13}$

The salient features are immediately apparent with the excitations separating in to discrete energy levels. The inclusion of $J_{n}^{\prime}$ is critical. Were there no interaction across the nonmagnetic Y layers, the energies of these modes would be independent of $q$. The levels are not flat, due to the magnetic interactions across the $\mathrm{Y}$ layers. All the energy levels are periodic functions of $q$ with a period of $2 \pi / \Lambda$ where $\Lambda$ is the thickness of a Dy/Y bilayer. The lowest energy mode has minima at $q=2 n \pi / \Lambda$, where $n$ is an integer. The inelastic structure factor is large at a number of these minima, predicting the appearance of superlattice peaks, however the model has a unique Brillouin zone center at $q=0$. The magnitudes of the structure factors are asymmetric around $q=0$ because the magnetic helix has different wave numbers in Dy and $\mathrm{Y}^{4}$

While being a distinctly different theoretical treatment, the net picture resembles so-called "Brillouin-zone folding" from the extra periodicity imposed by the multilayer structure. Discrete spin wave modes have been observed in confined systems, ${ }^{19}$ and "Brillouin-zone folding" has been reported for phonons ${ }^{20}$ and in plasmonic crystals. ${ }^{21}$ These observations could also be described by analogy with the theory presented here.

The DMI was not used to calculate the simulated data in Fig. 1, however it was used to model the experimental data. The helimagnetic structure along [0001] may be considered to be broken in to chiral domains. The characteristic lengths of these domains may be written as $L_{1}$ and $L_{2}$, thus the sample will have a net chiral polarization of

$$
\gamma=\frac{L_{1}-L_{2}}{L_{1}+L_{2}} .
$$

The DMI shows that the propagating spin excitations will see different potentials in different domains, depending on the chiral handedness of the domain. ${ }^{22,23}$ The energy difference between the potentials is $E_{\mathrm{DMI}} \propto|\mathbf{D}|$. The excitations will thus have different wave vectors, given by $\mathbf{k}_{1}$ and $\mathbf{k}_{2}$, and $E_{\mathrm{DMI}}$ may be written

$$
E_{\mathrm{DMI}} \propto \hbar \omega_{q}\left(1-\frac{\left|\mathbf{k}_{2}\right|}{\left|\mathbf{k}_{1}\right|}\right),
$$

where $\omega_{q}$ is the calculated spin wave frequency without the DMI. The excitations are thus subjected to a rescaling of energy following the equation:

$$
\hbar \omega_{q}^{\prime} \propto \frac{\frac{1-\gamma}{2} E_{\mathrm{DMI}}}{1-\frac{1-\gamma}{2 \hbar \omega_{q}} E_{\mathrm{DMI}}}+\hbar \omega_{q}
$$

\section{EXPERIMENTAL RESULTS AND DISCUSSION}

The sample used for the study had the form: $\mathrm{Y}_{50 \mathrm{~nm}}\left[\mathrm{Dy}_{4.3 \mathrm{~nm}} / \mathrm{Y}_{2.8 \mathrm{~nm}}\right]_{\times 350} / \mathrm{Y}_{234 \mathrm{~nm}} / \mathrm{Nb}_{200 \mathrm{~nm}} / \mathrm{Al}_{2} \mathrm{O}_{3}$ (substrate). It was grown by molecular beam epitaxy techniques with the crystallographic $c$ axis of the hcp rare-earths parallel with the surface normal. The surface area of the $\mathrm{Al}_{2} \mathrm{O}_{3}$ substrate was $30.5 \mathrm{~mm} \times 24.5 \mathrm{~mm}$, and the total mass of the magnetic rare earth was estimated to be $\sim 10 \mathrm{mg}$. The sample was also used in a recent study on field-induced chirality. ${ }^{6}$

Neutron-diffraction data from the sample are shown in Fig. 2, being measurements as a function of temperature along the [0001] direction. The data are typical for superlattices, with multiple Bragg peaks, and the structural quality is very similar to those quoted in other articles. ${ }^{4}$ The peak at neutron momentum transfer $Q \approx 2.21 \AA^{-1}$, which is visible at all temperatures, corresponds to the mean $c$-axis lattice parameter for Dy and Y, and is labeled $\langle N\rangle$. The peaks labeled $m_{H}$ correspond to the helimagnetic satellites of the Dy/Y (0002) and these correspond to $q=0$ in Fig. 1 while the magnetic peaks due to the superlattice periodicity are labeled $m_{S L}$. These vary with temperature as the magnetic turn angle and the mean magnetization have temperature dependence. ${ }^{4,5}$ Also shown is the $(11 \overline{2} 0)$ Bragg peak from the $\mathrm{Al}_{2} \mathrm{O}_{3}$ substrate. The data show that there is essentially no change in the magnetic structure for $T \leq 75 \mathrm{~K}$.

The neutron inelastic measurements were concentrated around $1.8 \leq Q \leq 2.0 \AA^{-1}$. This range contains a strong helical Bragg peak at $Q \approx 1.97 \AA^{-1}$, and is relatively free of strong elastic scattering which would add to background. Working at smaller $Q$ helped to avoid kinematic constraints and instrument hard limits, and also offered a gain in intensity due to the magnetic form factor.

Representative data showing a series of constant- $Q$ scans are shown in Fig. 3(a). For these measurements, IN14 was 


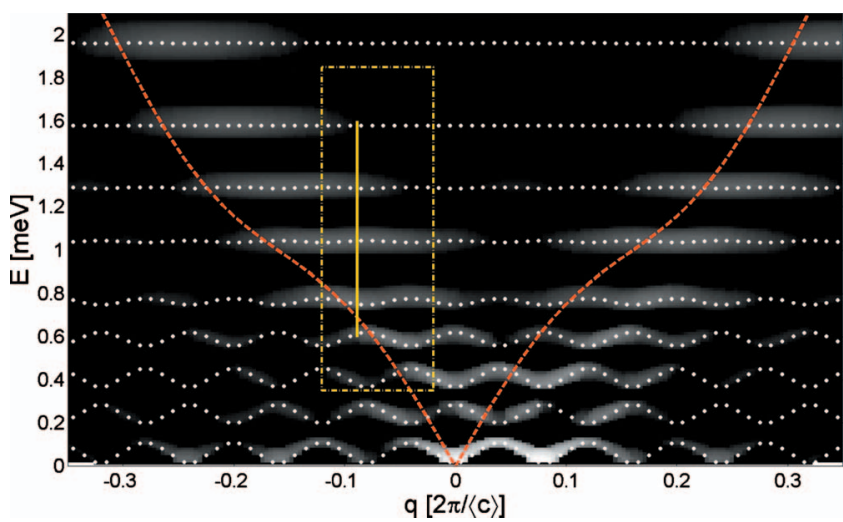

FIG. 1. (Color) A theory calculation showing part of the neutron inelastic structure factor from a $\mathrm{Dy}_{4.3 \mathrm{~nm}} / \mathrm{Y}_{2.8} \mathrm{~nm}$ superlattice as a function of the reduced reciprocal lattice vector, $q$. The energies of the discrete modes are shown by the white dotted lines with the shading representing the amplitudes. For clarity, the Bose factor and the DMI have not been included in the calculation. Also shown is the dispersion for bulk Dy (red, dashed lines) for comparison. The box and line show the regions and scans that correspond to the measurements in Figs. 3 and 4.

configured in the " $W$ " configuration with a fixed final wavenumber of $k_{f}=1.5 \AA^{-1}$. The monochromator and analyzer were vertically focused. No collimation was used before the sample and a $60^{\prime}$ collimator was used between sample and analyzer. The elastic energy full width for the measurements was $\sim 0.13 \mathrm{meV}$.

The measurements were made at $75 \mathrm{~K}$ because, as shown in Fig. 2, the magnetic structure is unchanged from the ground state, while the inelastic signal will be much larger due to the Bose factor. Figure 3(a) shows a clear dispersive signal merging with the tail of the helimagnetic peak at $Q$ $=1.968 \AA^{-1}$. This signal is not observed in measurements at $3 \mathrm{~K}$, where the inelastic intensity is very small due to the Bose factor, or at $300 \mathrm{~K}$, which is above the Néel temperature. Hence, the data show the scattering from magnetic ex-

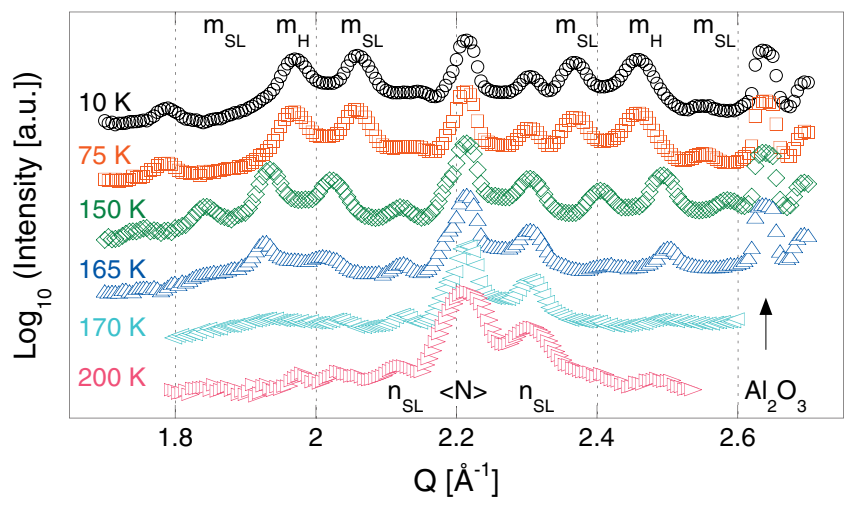

FIG. 2. (Color) Neutron-diffraction data as a function of the temperature measured on a Dy/Y superlattice. The peak corresponding to the mean $c$-axis lattice parameter is labeled $\langle N\rangle$ and the structural superlattice peaks are labeled $n_{S L}$. The magnetic satellites for this peak are labeled $m_{H}$. The magnetic peaks corresponding to the superlattice structure are labeled by $m_{S L}$. Also labeled is the Bragg peak corresponding to the $\mathrm{Al}_{2} \mathrm{O}_{3}$ substrate.

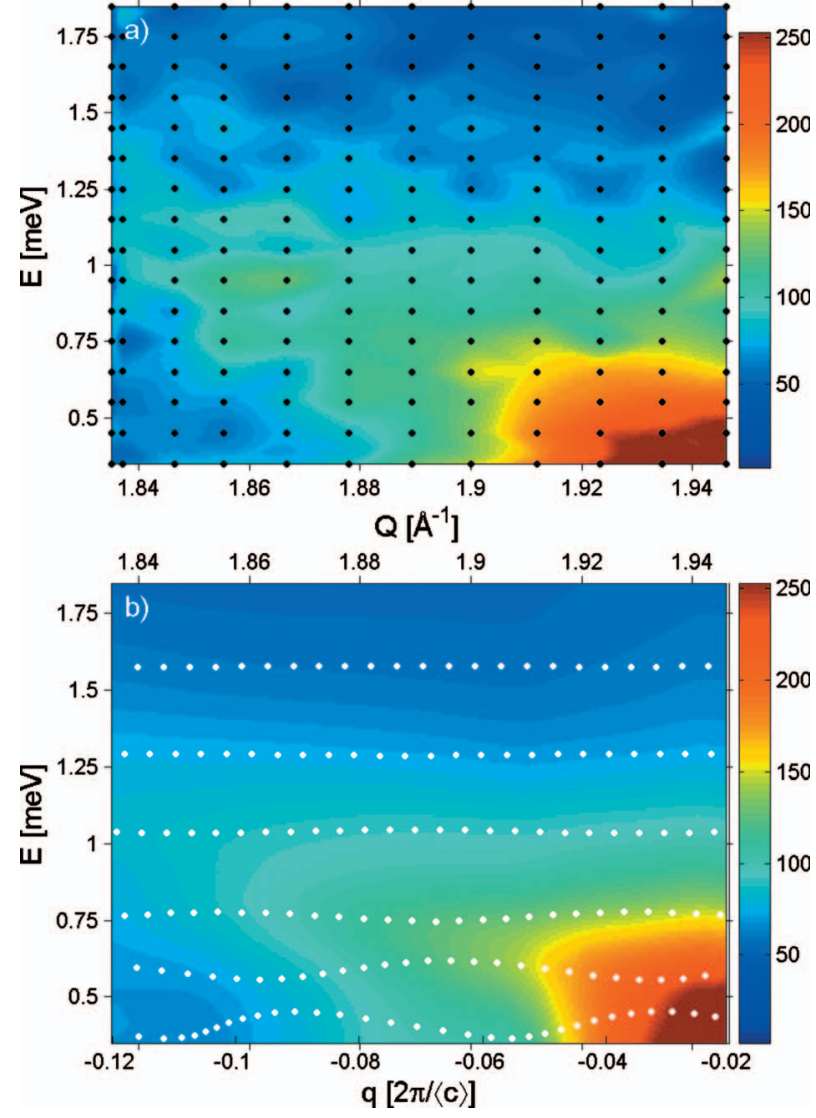

FIG. 3. (Color) (a) Neutron inelastic data near the Brillouinzone center obtained from the Dy/Y superlattice, measured at $75 \mathrm{~K}$. The measured time per point was $\sim 23 \mathrm{~min}$. The black dots indicate the measured data points. (b) Calculations of the corresponding neutron inelastic structure factor accounting for instrumental resolution, the DMI, background, and the intrinsic energy width of Dy spin excitations. The dotted lines show the energies of the discrete modes.

citations, and we attribute the peak at $Q=1.968 \AA^{-1}$ to be a Brillouin zone center. The data therefore correspond to the region of $(q, \omega)$ marked out by the box in Fig. 1 .

Three additional effects had to be applied to the model in Fig. 1 in order to achieve a satisfactory comparison between theory and experiment. First, the model was convoluted with the instrument resolution, which was calculated using RESLIB software in the "Popovici" mode ${ }^{24}$ and calibrated against the energy width of the incoherent elastic scattering. Second, the discrete modes in the model were converted to Lorentzian line shapes and broadened by a half-width half-maximum of $\Delta=0.18 \mathrm{meV}$. This corresponds to the width of the spin excitations in bulk Dy, ${ }^{14}$ and may be considered to be intrinsic. The DMI in Dy/Y superlattices gives an important contribution which, by Eq. (9), shifts the energies of the excitations, and this represents the third effect. This sample is known to have an imbalance of the population of chiral domains in zero field, parameterized by $\gamma=-0.1 .^{6}$ Based on literature, ${ }^{25-27}$ an energy estimate of $E_{\mathrm{DMI}}=0.25 \mathrm{meV}$ was included, resulting in the satisfactory agreement between data and theory shown in Fig. 3.

With the broadening of the excitations many of the features, namely, the discrete nature of the energy levels, be- 
come obscured. Indeed, the intrinsic width appears to cause the excitations to become some seven times larger than the instrumental resolution, estimated from the measured width of the incoherent elastic scattering. In an attempt to resolve two peaks at one $q$, measurements with higher resolution were made. Figure 4 shows high resolution data measured at $Q=1.87 \AA^{-1}$. IN14 was configured with a horizontally focused analyzer with $k_{f}=1.05 \AA^{-1}$, and with $60^{\prime}$ collimation before the sample. The elastic energy full width was $\sim 0.04 \mathrm{meV}$, representing an improvement in resolution of more than a factor of three over the data shown in Fig. 3(a). Under these conditions, data for $E<0.6 \mathrm{meV}$ could not be measured due to kinematic constraints. Data at $3 \mathrm{~K}$, where the observed intensity is very small due to the Bose factor, are included. These data act as a "quasibackground." It is worth reiterating that the signal was not visible at $300 \mathrm{~K}$ hence the inelastic signal at $75 \mathrm{~K}$ is indeed magnetic.

To compare with the intrinsic peak width of the Dy/Y superlattice, a similar inelastic measurement of an epitaxial Dy film is also shown in Fig. 4. The film had a thickness of $1 \mu \mathrm{m}$, which corresponds to $2 / 3$ the summed thickness of Dy in the Dy/Y sample. These measurements were made on IN12 with a horizontally focused analyzer, $k_{f}=1.3 \AA^{-1}$, and $60^{\prime}$ collimation before the sample. The elastic energy full width was $\sim 0.1 \mathrm{meV}$. Elastic scattering measurements showed that the magnetic structure of the film was the same as bulk. Figure 4 shows data measured at $Q=1.95 \AA^{-1}$, thus these data and the Dy/Y data correspond to $q \approx-0.09 \AA^{-1}$. Data are shown at $98 \mathrm{~K}$, in the helimagnetic phase, and at $300 \mathrm{~K}$, where the sample is paramagnetic. The data at $300 \mathrm{~K}$ are flat and featureless and thus represent a "background." The data at $98 \mathrm{~K}$ show a clear inelastic signal which appears at the correct energy for bulk Dy. After correcting for the instrument resolution, the measured Lorentzian half-width half-maximum was found to be $0.18 \pm 0.01 \mathrm{meV}$, which confirms the previously published data for bulk Dy. ${ }^{14}$

The feature in the Dy/Y data in Fig. 4 is very broad in energy. It is much broader than the feature in the Dy data, even though the instrumental resolution is better. Furthermore, it shows some energy-dependent structure. The data are modeled by the theory which predicts modes, centered at the arrows in Fig. 4, whose amplitudes are given by the calculated inelastic structure factor and whose widths are the experimentally measured intrinsic width. The match between theory and experiment is excellent, thus the data show evidence for multiple peaks.

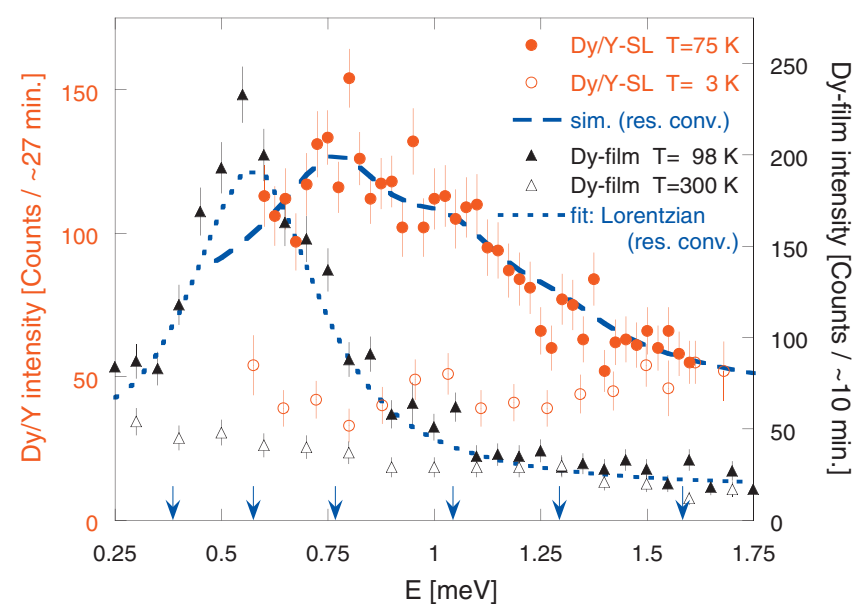

FIG. 4. (Color) An overlay of high-resolution inelastic data measured at the corresponding positions in the Brillouin zone for the Dy/Y superlattice (Dy/Y-SL) and a Dy film (Dy-film) at IN14 and IN12, respectively. The red data correspond to the red scale and the black data to the black scale. Shown in blue are simulations and fits for the intensities. The arrows mark the energies of the discrete modes for the Dy/Y-SL.

\section{CONCLUSIONS}

The experiments show solid evidence for the formation of discrete energy modes in Dy/Y superlattices and the observation of multiple peaks at a single $q$ in an inelastic neutron scan. The results show that it is possible to use neutron scattering to investigate the spin dynamics of nanostructured magnetic systems in wide regions of the Brillouin zone. The observations are quantitatively reproduced by the theory, and the significance of the DMI interaction, in addition to the RKKY interaction, is shown for the spin-wave spectra. The presence of multiple peaks raises the possibility of treating magnetic superlattices such as band-gap magnonic crystals, which are expected to show great promise in applications such as magnetoelectronic devices. ${ }^{28,29} \mathrm{New}$, higher flux neutron sources and the next generation of improved instrumentation will allow the study of technologically relevant nanostructures where the experimental access to the spin dynamics is essential.

\section{ACKNOWLEDGMENTS}

The authors would like to thank A. N. Bogdanov, M. Rotter, and D. Lott for useful discussions.

\footnotetext{
*wildes@ill.fr

${ }^{1}$ J. Jensen and A. R. Mackintosh, Rare Earth Magnetism: Structures and Excitations (Clarendon Press, Oxford, 1991).

${ }^{2}$ R. A. Cowley and J. Jensen, J. Phys.: Condens. Matter 4, 9673 (1992).

${ }^{3}$ J. Kwo, in Thin Film Growth Techniques for Low-Dimensional Structures, edited by R. F. C. Farrow, S. S. P. Parkin, P. J. Dobson, J. H. Leaves, and A. S. Arrott (Plenum, New York, 1987).
}

${ }^{4}$ R. W. Erwin, J. J. Rhyne, M. B. Salamon, J. Borchers, S. Sinha, R. Du, J. E. Cunningham, and C. P. Flynn, Phys. Rev. B 35, 6808 (1987).

${ }^{5}$ C. F. Majkrzak, J. Kwo, M. Hong, Y. Yafet, D. Gibbs, C. L. Chien, and J. Bohr, Adv. Phys. 40, 99 (1991).

${ }^{6}$ S. V. Grigoriev, Y. O. Chetverikov, D. Lott, and A. Schreyer, Phys. Rev. Lett. 100, 197203 (2008).

${ }^{7}$ A. Schreyer, T. Schmitte, R. Siebrecht, P. Bödeker, H. Zabel, S. 
H. Lee, R. W. Erwin, C. F. Majkrzak, J. Kwo, and M. Hong, J. Appl. Phys. 87, 5443 (2000)

${ }^{8}$ R. E. Camley, J. Magn. Magn. Mater. 200, 583 (1999) and references therein.

${ }^{9}$ L. L. Hinchey and D. L. Mills, Phys. Rev. B 33, 3329 (1986).

${ }^{10}$ J. G. LePage and R. E. Camley, Phys. Rev. Lett. 65, 1152 (1990).

${ }^{11}$ N. N. Chen and D. Y. K. Ko, J. Phys.: Condens. Matter 5, 3463 (1993).

${ }^{12}$ A. Yoshimori, J. Phys. Soc. Jpn. 14, 807 (1959).

${ }^{13}$ R. M. Nicklow, N. Wakabayashi, M. K. Wilkinson, and R. F. Reed, Phys. Rev. Lett. 26, 140 (1971).

${ }^{14}$ R. M. Nicklow, J. Appl. Phys. 42, 1672 (1971).

${ }^{15}$ Y. Yafet, J. Appl. Phys. 61, 4058 (1987).

${ }^{16}$ Y. Yafet, J. Kwo, M. Hong, C. F. Majkrzak, and T. O'Brien, J. Appl. Phys. 63, 3453 (1988).

${ }^{17}$ R. E. Camley, J. Kwo, M. Hong, and C. L. Chien, Phys. Rev. Lett. 64, 2703 (1990).

${ }^{18}$ W. Marshall and S. W. Lovesey, Theory of Thermal Neutron Scattering (Clarendon, Oxford, 1971).

${ }^{19}$ S. O. Demokritov and B. Hillebrands, in Spin Dynamics in Confined Magnetic Structures I, edited by B. Hillebrands and K. Ounadjela (Springer, Berlin, 2002), pp. 65-92.
${ }^{20}$ A. Magerl and H. Zabel, Phys. Rev. Lett. 46, 444 (1981); J. R. Dutcher, S. Lee, B. Hillebrands, G. J. McLaughlin, B. G. Nickel, and G. I. Stegeman, ibid. 68, 2464 (1992).

${ }^{21}$ V. M. Muravev, A. A. Fortunatov, I. V. Kukushkin, J. H. Smet, W. Dietsche, and K. von Klitzing, Phys. Rev. Lett. 101, 216801 (2008).

${ }^{22}$ A. N. Bogdanov and U. K. Rössler, Phys. Rev. Lett. 87, 037203 (2001).

${ }^{23}$ M. Bode, M. Heide, K. von Bergmann, P. Ferriani, S. Heinze, G. Bihlmayer, A. Kubetzka, O. Pietsch, S. Blugel, and R. Wiesendangere, Nature (London) 447, 190 (2007).

${ }^{24}$ http://www.neutron.phys.ethz.ch/Resources/Reslib/index.html; email: zhelud@ethz.ch

${ }^{25}$ A. Zorko, S. Nellutla, J. van Tol, L. C. Brunel, F. Bert, F. Duc, J. C. Trombe, M. A. de Vries, A. Harrison, and P. Mendels, Phys. Rev. Lett. 101, 026405 (2008).

${ }^{26}$ A. Zorko, D. Arcon, H. van Tol, L. C. Brunel, and H. Kageyama, Phys. Rev. B 69, 174420 (2004).

${ }^{27}$ P. A. Lindgård, Phys. Rev. B 17, 2348 (1978).

${ }^{28}$ Z. K. Wang, V. L. Zhang, H. S. Lim, S. C. Ng, M. H. Kuok, S. Jain, and A. O. Adeyeye, Appl. Phys. Lett. 94, 083112 (2009).

${ }^{29}$ M. Krawczyk and H. Puszkarski, Phys. Rev. B 77, 054437 (2008). 